\title{
Effects of different sutures on fibrosis and wound healing in a rabbit model of corneal wounds
}

\author{
YING LI ${ }^{1}$, HUI J. CHEN ${ }^{1}$, HUA ZHANG ${ }^{2}$, JIAN G. WU ${ }^{1}$, YUN T. HU ${ }^{1}$ and ZHI Z. MA ${ }^{1}$ \\ Departments of ${ }^{1}$ Ophthalmology (Eye Center) and ${ }^{2}$ Statistics, \\ Peking University Third Hospital, Beijing 100191, P.R. China
}

Received January 14, 2016; Accepted July 20, 2016

DOI: $10.3892 /$ etm.2016.3703

\begin{abstract}
The aim of the study was to investigate wound healing and scar formation in rabbit corneal lamellar wounds repaired with simple interrupted sutures (SIS) or horizontal mattress sutures (HMS). Two parallel 'I'-shaped lamellar cornea wounds were created in one eye of 40 white New Zealand rabbits, while 5 uninjured rabbits were sacrificed to serve as normal controls. One side of the wounds, in the test rabbits, was closed with SIS, while the other side was treated with HMS. Ten days later, the stitches were removed under anesthesia. The animals were sacrificed on days 14 and 21, and months 3 and 6 after the suturing surgery, and corneal samples were subjected to histological and immunofluorescent studies: $\alpha$-smooth muscle actin ( $\alpha$-SMA) and vimentin were used to detect myofibroblasts and fibroblasts, respectively, and collagen type I and III was used to detect extracellular matrix (ECM) deposition. Relevant mRNA levels were assessed by quantitative polymerase chain reaction (qPCR) to elucidate the differences in wound healing and formation of fibrosis. Macroscopic and hematoxylin and eosin staining observations showed that the two sides of the wounds developed the most prominent fibrotic tissue on day 21 . The immunofluorescence and qPCR results showed that HMS wounds produced increased $\alpha$-SMA, vimentin and collagen type III compared to the SIS wounds on day 14 or 21 . The collagen type I expression showed no distinctive difference in SIS and HMS wounds. In conclusion, corneal lamellar wounds treated with SIS developed less fibrotic-related proteins and related mRNA in the early stages of wound healing than wounds treated with HMS. Although differences were not distinct after 3 months, the results of the present study suggest a benefit in choosing SIS over HMS, as at least the initial fibrotic process seems more benign with SIS. Corneal wounds should be carefully sutured, ensuring the tissue is well aligned.
\end{abstract}

Correspondence to: Dr Zhi Z. Ma, Department of Ophthalmology (Eye Center), Peking University Third Hospital, 49 Huayuan North Road, Beijing 100191, P.R. China

E-mail: xnxeiixh93275@163.com

Key words: cornea, wound healing, sutures, fibrosis

\section{Introduction}

It is generally acknowledged that, whenever indicated, wounds should be carefully sutured (1). The simple interrupted suture (SIS) and horizontal mattress suture (HMS) are two common wound closure methods. SIS is used as the wound is kept clean and smooth, and it aligns tissues well. HMS is chiefly used in big tension wounds, in repair of blood vessel rupture or in closing the peritoneum, to create a smooth inner wall $(2,3)$. However, the differences in the resulting fibrotic processes of the two types of suture remain to be clarified. Thus, a self-comparison model was formed to examine the different fibrotic processes of the two sutures in corneal wound healing.

Since the cornea is transparent, avascular and composed of simple substances, it is an ideal site to investigate the process of wound healing (2). When the cornea is injured, the quiescent keratocytes in the wound stroma become apoptotic, and the keratocytes nearby are activated. The activated keratocytes proliferate and differentiate into spindle-shaped fibroblasts that migrate towards the wound area (4). After arriving at the wound edge, some of these cells enlarge and differentiate into myofibroblasts within the stroma (5). Myofibroblasts fill the wound in an interwoven and interconnected network pattern, and express filaments of $\alpha$-smooth muscle actin ( $\alpha$-SMA) to promote wound contraction (6). Myofibroblasts are less translucent than static keratocytes and they further decrease stromal transparency by altering the organization and composition of the extracellular matrix (ECM) (7). Vimentin is one of the many intermediate filaments of mesenchymal cells such as fibroblasts and endothelial cells (8). Vimentin is needed during tissue repair and its overexpression plays an unappreciated pathogenic role in corneal fibrotic conditions (9). Fibroblasts and myofibroblasts synthesize the ECM components, including collagen types I and III, and fibronectin (10). These proteins are involved in the fibrosis of wound healing.

In the present study, we utilized a rabbit model to test the hypothesis that the two types of sutures differ in the fibrotic processes they stimulate.

\section{Materials and methods}

Animals. In total, 45 healthy male New Zealand white rabbits (Peking University Health Science Center, Beijing, China), weighing 2.0-2.5 kg, were used in the present study. 
Five rabbits were sacrificed as normal controls. The remaining 40 rabbits were divided into 4 groups $(\mathrm{n}=10)$ and were sacrificed at 14 days, 21 days, 3 months and 6 months after the initial incision. The present study was conducted in strict accordance with the recommendations in the Guide for the Care and Use of Laboratory Animals of the National Institutes of Health. The Institutional Animal Care and Use Committee of Peking University approved the animal protocol (permit no. LA2012-54).

Surgeries were performed under general and topical anesthesia. General anesthesia was induced with a muscle injection of ketamine $\mathrm{HCl}(0.1 \mathrm{ml} / \mathrm{kg})$ and Su-Mian-Xin $(0.1 \mathrm{ml} / \mathrm{kg})$. Topical anesthesia was applied with Benoxil. Efforts were made to minimize suffering.

Animal surgery. The surgeries were randomized to the left or right eye of the rabbits, but surgery was performed to only one eye for each rabbit. The surgical procedure was as follows: After sterilization of the palpebrae and the conjunctival sac, the palpebrae were opened by use of a spatula, and the superior and inferior recti muscles were stabilized with 5-0 sutures. Two 'I'-shaped incisions on each side of the cornea, parallel to the corneal median vertical line were made. The lengths of the top and bottom ends of the vertical wounds were $3 \mathrm{~mm}$, the vertical length of the wound was $6 \mathrm{~mm}$, and the incision depth was $1 / 2$ of the corneal thickness. The vertical flaps to the left and right were separated $1.5 \mathrm{~mm}$ through the lamellar stroma. After washing the wounds with $0.9 \%$ normal saline, $0.2 \mathrm{ml}$ of aqueous was aspirated from the anterior chamber with a $1.0 \mathrm{ml}$ syringe at the vertical limbus position to reduce the corneal tension. The SIS wound was closed with five interrupted 8-0 silk sutures (Snake, Germany), while the HMS wound was closed with three interrupted horizontal mattress 8-0 silk sutures (Fig. 1E). After finishing the sutures, $0.5 \mathrm{ml}$ tobramycin was injected into the subconjuctival space, and erythromycin ointment was applied to the conjunctival sac. Ofkoxacin eye drops were administered into the wounded eye 3 times per day for 1 month. Ten days after the surgery, the sutures were removed under anesthesia. The animals were sacrificed in a randomized manner on days 14 and 21, and months 3 and 6 after surgery.

Regular images. Photos were obtained at 14 and 21 days, and at 3 and 6 months after surgery under general and topical anesthesia. The regular images captured were obtained using a Topcon OMS 800 Surgery Microscope with an NC Video 1.0 system (Newcom Technology Co., Ltd., Shenzhen, China).

Tissue processing. In total, 50 corneas were harvested, including 40 corneas from the four treated groups $(n=10)$, and 10 corneas from the five blank control rabbits once they were sacrificed. Half of the corneas in each group were used for detection of immunofluorescence, while the remaining $50 \%$ of the corneas were used for quantitative polymerase chain reaction (qPCR) analysis. The corneas used for immunofluorescence were fixed in $4 \%$ paraformaldehyde (PFA) immediately after death. After $1 \mathrm{~h}$, the corneas were harvested from the eyeballs and were placed in 4\% PFA solution for $24 \mathrm{~h}$ at $4^{\circ} \mathrm{C}$. After fixation, the corneas were washed twice in $1 \mathrm{X}$ phosphate-buffered saline (PBS) for $30 \mathrm{~min}$. The corneas were

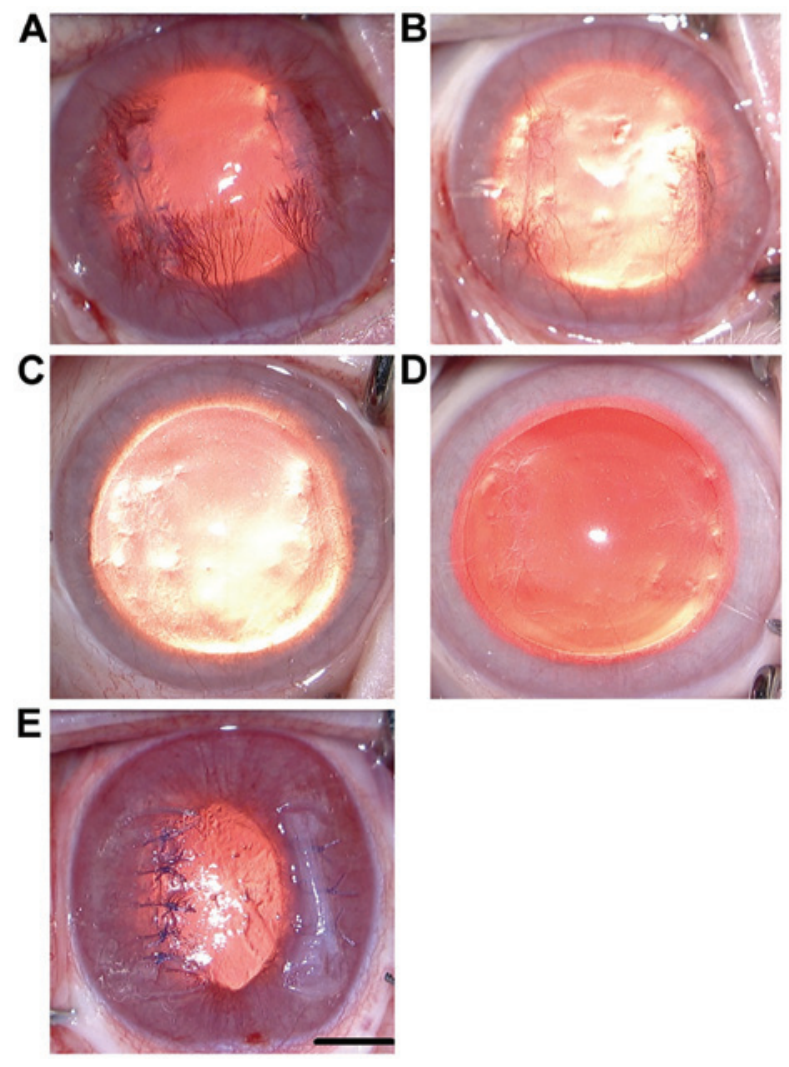

Figure 1. Regular images of corneal wounds after surgery. (A-E) Wounded corneas at different time points, i.e., (A) 14 days, (B) 21 days, (C) 3 months, (D) 6 months and (E) right after surgery. The left wound in each image was repaired with simple interrupted sutures, while the right wounds were repaired with horizontal mattress sutures. The scars of the two wounds were most different on day 21. Bar equals $3 \mathrm{~mm}$.

then dehydrated in a $30 \%$ sucrose solution, embedded into OCT (Sakura Finetek, Torrance, CA, USA), frozen in liquid nitrogen for $3 \mathrm{~min}$ and preserved in a refrigerator at $-80^{\circ} \mathrm{C}$. The prepared samples were cut into $7 \mu \mathrm{m}$ sections using a Leica Freezing Microtome (Nussloch, Germany) 1 day prior to the subsequent procedures. The frozen sections were immersed in ice-cold acetone for $10 \mathrm{~min}$ prior to drying in air for $15 \mathrm{~min}$, and were kept in a $-80^{\circ} \mathrm{C}$ refrigerator for immunofluorescence detection on the following day.

The central corneas of the eyeballs used for qPCR were marked with a $12-\mathrm{mm}$ diameter trephine (Meisinger, Centennial, CO, USA) and excised with scissors to remove the rims. The obtained grafts were cut vertically in half and were preserved in labeled freezing tubes (Corning Inc., Acton, MA, USA) and kept at $-80^{\circ} \mathrm{C}$ until mRNA assessment. The primers used to amplify the five genomic sequences are shown in Table I.

Immunofluorescence. Immunofluorescent staining was performed to detect $\alpha$-SMA, vimentin, and collagen types I and III, which are markers of myofibroblasts, fibroblasts, and new ECM protein deposition, respectively. Frozen sections were air dried and washed three times in 1X PBS for $5 \mathrm{~min}$. The sections were then blocked with goat serum for $1 \mathrm{~h}$ and repaired with 1X Frozen Sections Antigen Repair Agent (Beyotime Biotech, Jiangsu, China) for $5 \mathrm{~min}$ at room temperature. Subsequently, 1:100 primary antibodies 
were applied overnight in a moist chamber set at $4^{\circ} \mathrm{C}$. The following day, 1:300 diluted anti-mouse IgG secondary antibodies, conjugated with fluorescein, were applied at room temperature for $1 \mathrm{~h}$. After washing 3 times in PBS for $5 \mathrm{~min}$, DAPI Fluoromount-G (SouthernBiotech, Birmingham, AL, USA) was mounted for staining of the nuclei and the samples were covered with coverslips. The primary antibodies used were: monoclonal $\alpha$-SMA antibody (mouse anti-rabbit; dilution of 1:200; Sigma, St. Louis, MO, USA; cat. no. A5228), monoclonal vimentin antibody (mouse anti-rabbit, dilution of 1:500; Abcam, Cambridge, UK; cat. no. ab30436), collagen type I (monoclonal mouse anti-rabbit; dilution of 1:500; Abcam; cat. no. ab292), and collagen type III (monoclonal mouse anti-rabbit; Abcam; dilution of 1:500; cat. no. ab7778). The tissues were observed and photographed under a Leica fluorescence microscope camera (Leica DM3000). The negative controls were prepared using PBS, rather than primary antibodies.

Semi-quantitative PCR. The mRNA levels of a-SMA, collagen type I and III were analyzed by qPCR. Five corneal grafts of the rabbits that did not undergo surgery were used as negative controls to minimize the potentially confounding effects of natural variability in gene expression. Total RNA was extracted using an RNA purification kit (Invitrogen Life Technologies, Austin, TX, USA), according to the manufacturer's instructions. First-strand cDNA was synthesized using a synthesis kit (Avian Myeloblastosis Virus First-Strand cDNA Synthesis kit, Life Technologies, Carlsbad, CA, USA). The cDNA samples were then divided into tubes containing a PCR reaction mixture (Applied Biosystems Life Technologies, Foster City, CA, USA) with specific primers (Table I). For each treatment, a PCR reaction mixture was prepared, containing $1 \mu \mathrm{l}$ of cDNA, $10 \mu \mathrm{l}$ of PCR master mix (SYBR-Green; Applied Biosystems Life Technologies), $1 \mu \mathrm{l}$ of each primer and $7 \mu \mathrm{l}$ of $\mathrm{ddH}_{2} 0$ in a total volume of $20 \mu \mathrm{l}$. Semi-quantitative RT-PCR was performed with an StepOnePlus Real-Time PCR instrument (Applied Biosystems Life Technologies). The samples were assayed in triplicate. The cycling conditions were initiated at $95^{\circ} \mathrm{C}$ for 2 min, followed by 40 cycles consisting of a $10-\mathrm{sec}$ melting interval at $95^{\circ} \mathrm{C}$ and a 40 -sec interval for annealing at $60^{\circ} \mathrm{C}$. Immediately after the amplification, melt curve protocols were performed to ensure that primer-dimers and other non-specific products were minimized or eliminated. For data analyses, the cycle threshold (CT) of each gene for the surgery corneal grafts was normalized to the corresponding value for the control cornea and this value was used to calculate the fold-change with the $2^{-\Delta \Delta \mathrm{CT}}$ method. The results are presented as significant increases or decreases in mRNA detected in the experimental groups compared with their respective controls.

Statistical analysis. The corneal samples were analyzed individually (not pooled) $\geq 3$ times. The individual data values from each corneal sample were statistically analyzed using SPSS statistical software package, version 17.0 (SPSS, Inc., Chicago, IL, USA). The paired t-test was used to compare mRNA values between the two differentially sutured groups. One-way ANOVA was used to compare mRNA values of different time 

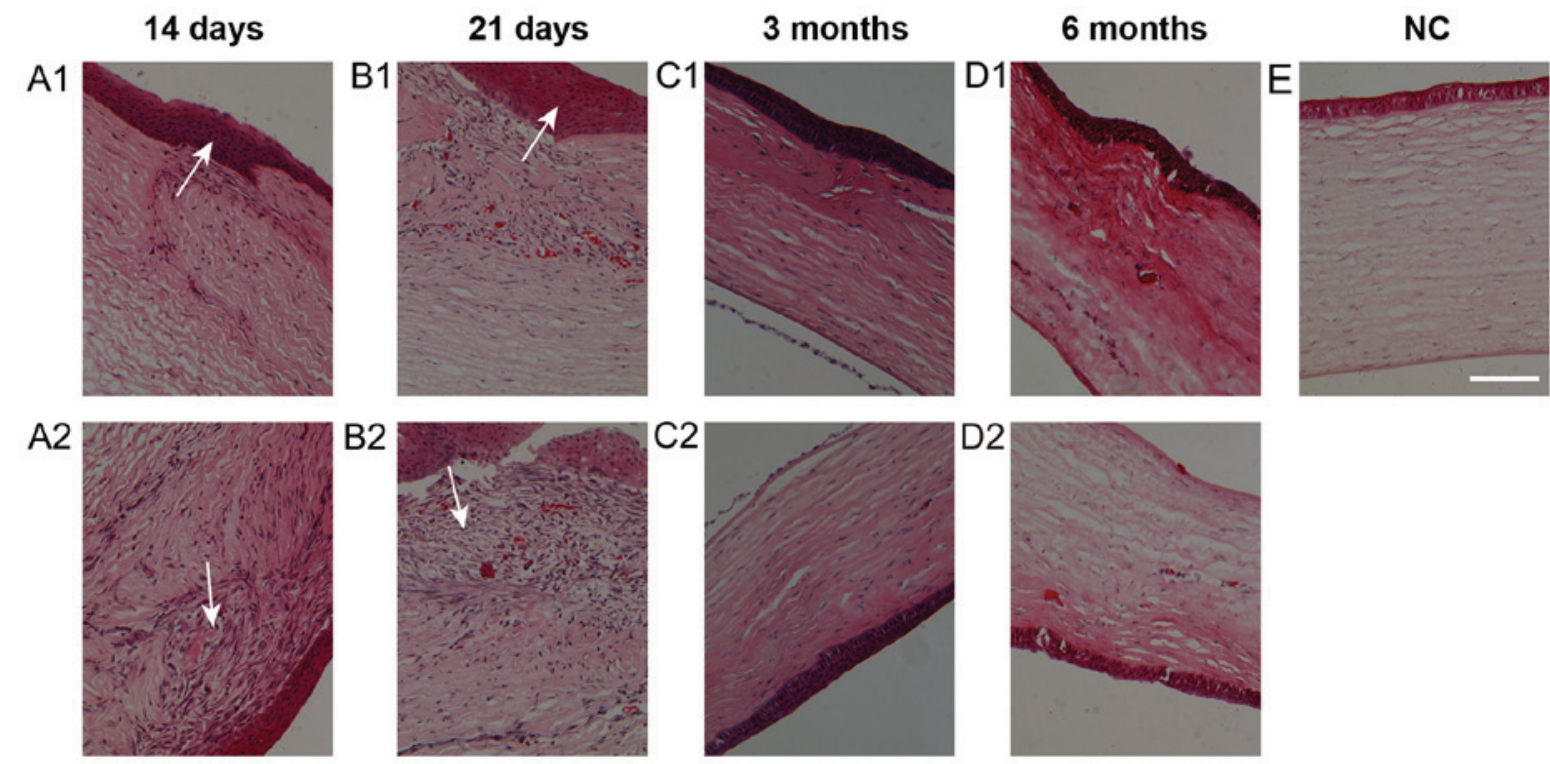

Figure 2. Hematoxylin and eosin (H\&E) staining of corneal wounds after surgery. (A1-D1) H\&E staining (x20 magnification) of the simple interrupted sutures wounds from frozen sections, (A2-D2) horizontal mattress sutures wounds. (E) Normal corneal section (NC). The arrows in (A1 and B1) show epithelial proliferation in wounds and those in (A2 and B2) show many fibrotic cells accumulated under the epithelium. Bar equals $100 \mu \mathrm{m}$.
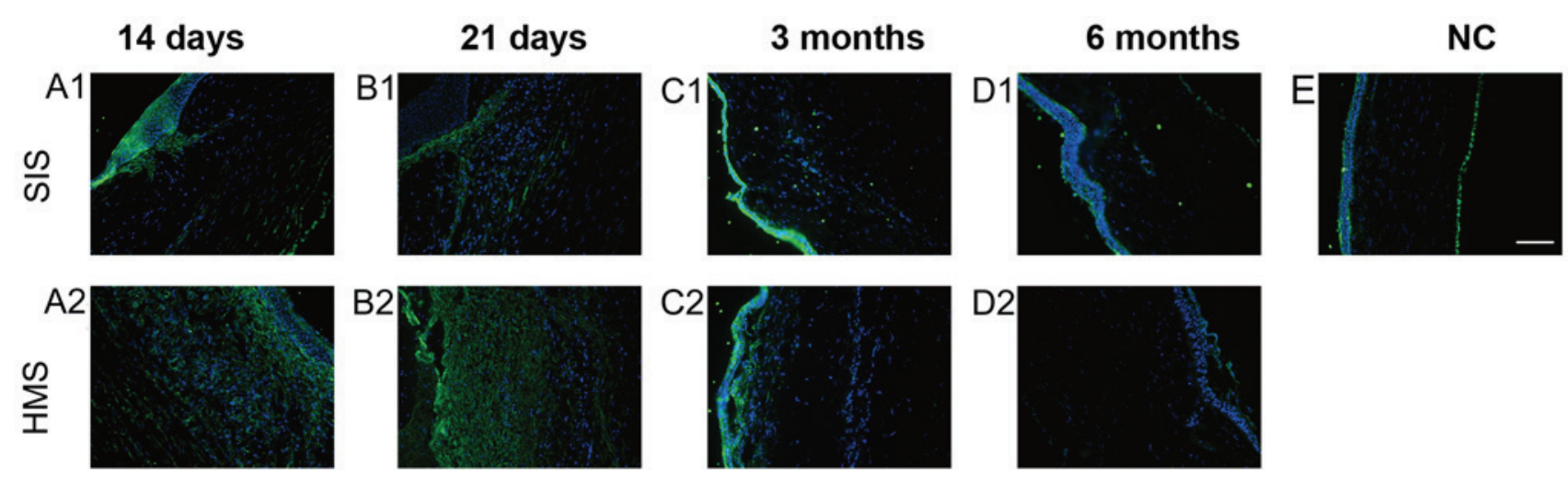

Figure 3. $\alpha$-smooth muscle actin ( $\alpha$-SMA) expression in corneal wounds after surgery. Green staining shows positive expression of $\alpha$-SMA, a marker of myofibroblasts. (A1-D1) Show the $\alpha$-SMA stained simple interrupted sutures (SIS) wounds; (A2-D2) show the $\alpha$-SMA stained horizontal mattress sutures (HMS) wounds. (E) Stands for normal control (NC). The staining of both wounds reached its peak on day 21 (B1 and B2), while it decreased dramatically at 3 months. The staining of HMS wounds was stronger than that of SIS wounds on days 14 and 21. Bar equals $100 \mu \mathrm{m}$.

points in each group. The statistical results with $\mathrm{P}<0.05$ was considered to indicate a statistically significant difference.

\section{Results}

Morphology results: Clinical observations of wound healing following corneal incisions closed with SIS or HMS. Images of clinical signs of the wound-healing process were captured. Fig. 1E shows an image directly after the procedures. SIS closure is shown on the left wound, while HMS closure is shown on the right wound. The neovascularization of wounds reached a peak on day 14 (Fig. 1A) and began to fade away. At 3 months, neovascular vessels and scarring tissue had virtually disappeared (Fig. 1C). A comparison revealed that the scars of HMS wounds were denser than those of SIS wounds at month 3 (Fig. 1C). Fibrosis of the two sides were apparent on day 21 (Fig. 1B). The scars appeared to show no apparent disparities between the two sides at month 6 (Fig. 1D).
Hematoxylin and eosin staining of the HMS and SIS wounds following surgery. The two types of sutured wounds appeared more cellular than normal corneas. The epithelial cells of the wounds showed proliferation and multilayered morphology at days 14 and 21 (Fig. 2A1, A2, B1 and B2). At 3 and 6 months after surgery, the fibrotic cells in the wound area had almost disappeared (Fig. 2C1, D1 and C2, D2). Three months after surgery, the fibrotic cells in the wound area had almost disappeared (Fig. 2C1 and C2). However, the hypercellular interwoven tissues under the epithelium in HMS wounds were more abundant than in the SIS wounds on days 14 and 21 (Fig. 2A1, A2, B1 and B2). The wounds on the two sides showed signs of normal recovery to almost normal corneal tissues (Fig. 2C-E).

Immunofluorescence. The $\alpha$-SMA-positive cells emerged in the wound areas on the two sutures and reached a peak at day 21 (Fig. 3B1 and B2). At 3 and 6 months, the $\alpha$-SMA 

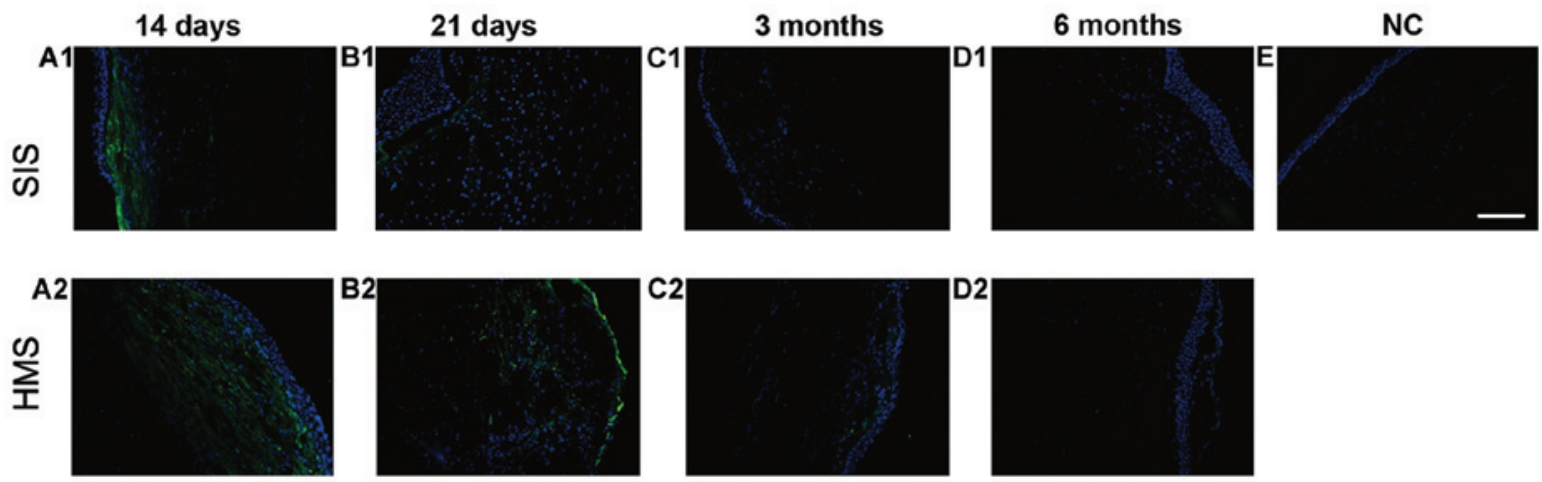

Figure 4. Vimentin expression in corneal wounds after surgery. Green staining shows a positive expression of vimentin, the marker of fibroblast cells. (A1-D1) Staining of simple interrupted sutures (SIS) wounds; and (A2-D2) staining of horizontal mattress sutures (HMS) wounds. (E) Staining of normal cornea (NC). On day 14, the positive expression of vimentin in the two wounds was distinctive. NC, normal control. Bars equal $100 \mu \mathrm{m}$.
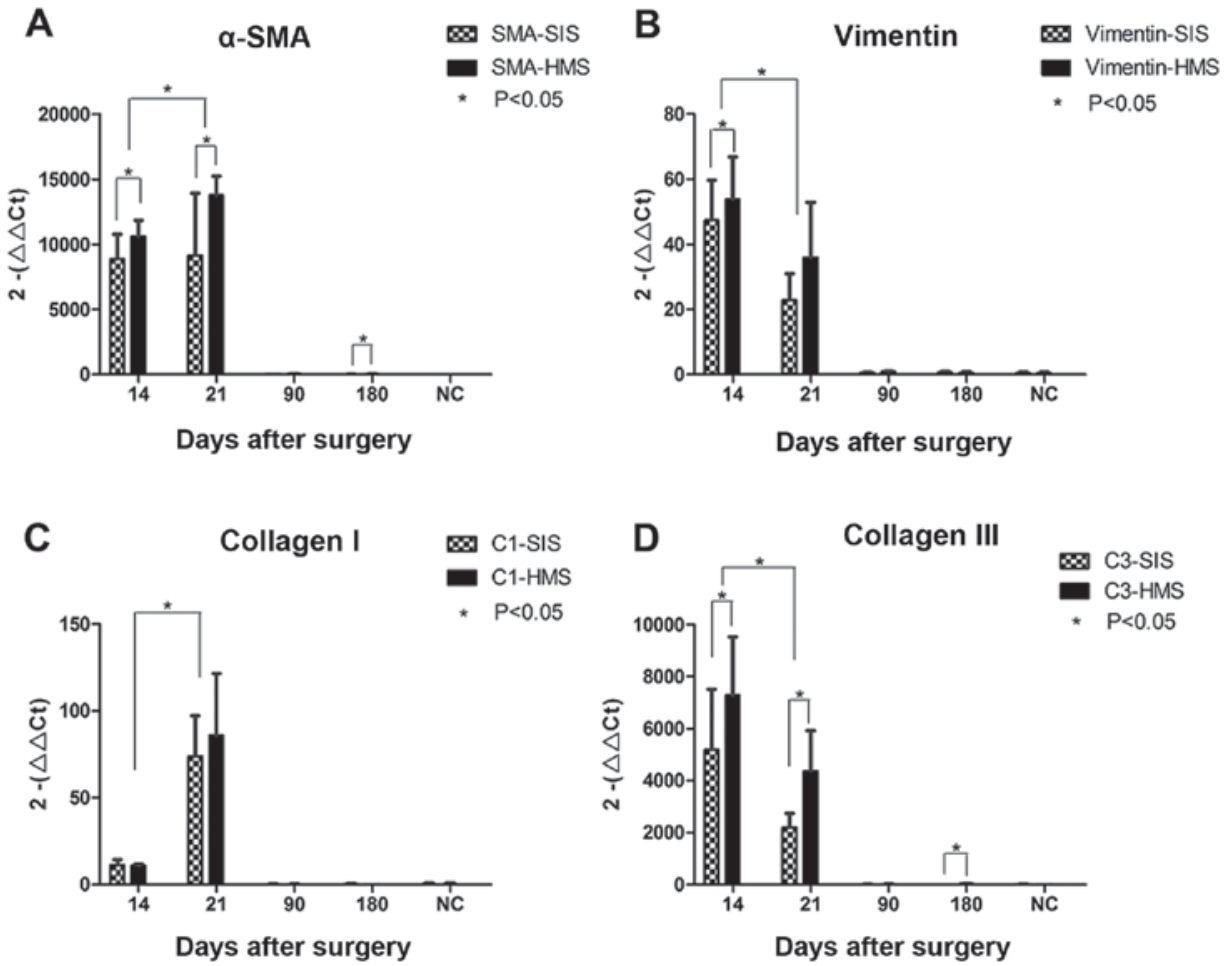

Figure 5. mRNA expression of $\alpha$-smooth muscle actin ( $\alpha$-SMA), vimentin, collagen types I and III in simple interrupted sutures (SIS) and horizontal mattress sutures (HMS) wounds after surgery. (A-D) mRNA expression of $\alpha$-SMA, vimentin, collagen types I and III in corneal wounds, separately. The mRNA expression of $\alpha$-SMA and collagen type III between SIS and HMS wounds was significantly different on days 14 and 21 (A and D). The mRNA expression of vimentin between SIS and HMS was significantly different on day 14. The collagen type I mRNA expression showed no difference between SIS and HMS wounds. ${ }^{*} \mathrm{P}<0.05$ of line-connected groups. NC, normal control.

staining on HMS wounds was much more intense than that on the SIS wounds on 21 day (Fig. 3B1 and B2). At 3 months, the $\alpha$-SMA-stained cells had nearly disappeared, except for the region immediately under the epithelium (Fig. 3C1, D1 and C2, D2). The analysis of control samples demonstrated this antibody reacts with stromal and endothelial cells in the normal cornea (Fig. 3E).

The number of vimentin-positive cells on both wounds was noted on days 14 and 21 (Fig. 4A1, A2, B1 and B2). At 3 and 6 months after surgery, the vimentin-positive cells were barely visible (Fig. 4C1, D1 and C2, D2). There was no positive staining in the normal cornea, indicating vimentin did not stain keratocytes (Fig. 4E). During the study, the peak of detection of the myofibroblast's marker $\alpha$-SMA was on day 21 (Figs. 3B1, B2 and 5A), which was later than that of the fibroblast marker vimentin. Vimentin expression reached its peak on day 14 (Figs. 4A1, A2 and 5B).

Collagen type I was found in the entire cornea except in the fibrotic region, especially on day 21 (Fig. 6B1 and B2). At that point the expression pattern of collagen type I resembled that of a ring with the center region full of fibrotic tissue containing collagen type III and $\alpha$-SMA. The normal cornea staining demonstrated collagen type I expression in the stroma (Fig. 6D). 


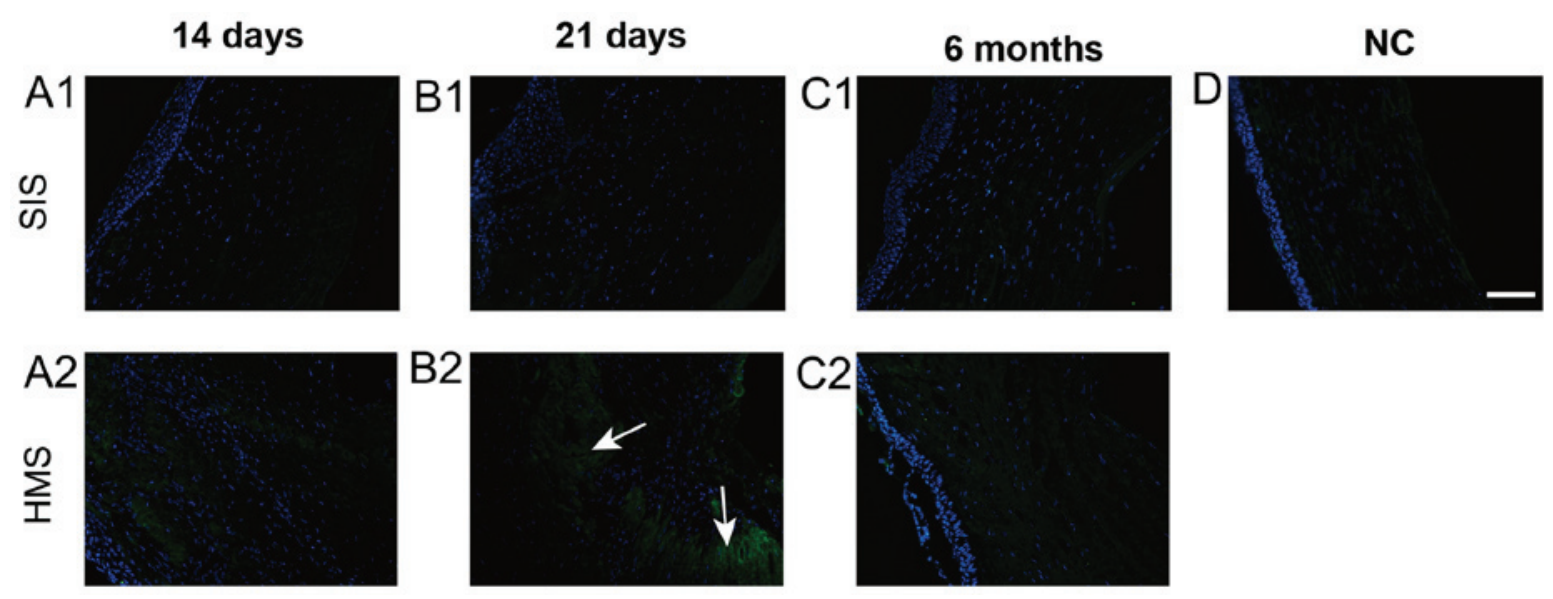

Figure 6. Collagen type I in corneal wounds after surgery. Positive expression of collagen type I is evident as green staining. (A1-C1) Staining of simple interrupted sutures (SIS) wounds, (A2-C2) staining of horizontal mattress sutures (HMS) wounds, and (D) staining of normal cornea (NC). Arrows indicate that the collagen type I expression resembles a 'ring' in the stroma on day $21 . \mathrm{NC}$, normal control. Bar equals $100 \mu \mathrm{m}$.

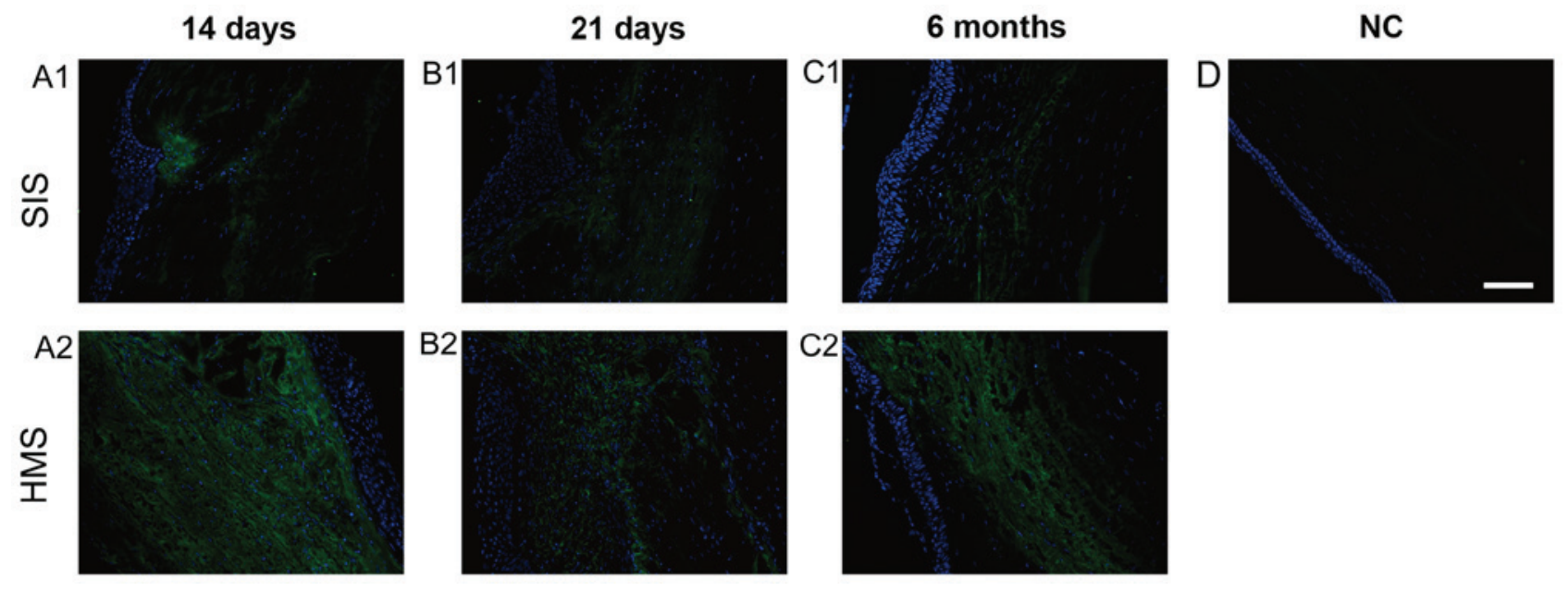

Figure 7. Collagen type III in corneal wounds after surgery. Positive expression of collagen type III is observed as green staining. (A1-C1) Staining of simple interrupted sutures (SIS) wounds, (A2-C2) staining of horizontal mattress sutures (HMS) wounds, and (D) staining of normal cornea (NC). The highest expression was evident on days 14 and 21 in both wounds. The expression in HMS wounds was higher than that in SIS wounds (A1-C1 and A2-C2). NC, normal control. Bar equals $100 \mu \mathrm{m}$.

The staining of collagen type III in HMS wounds was most intense on days 14 and 21 (Fig. 7A1, A2, B1 and B2). The expression of collagen type III was gradually decreased after 6 months (Fig. 7C1 and C2). Notably, the immunofluorescence of collagen type III in HMS wounds was much stronger than that in SIS wounds on day 21, and month 6 (Fig. 7A1-C1 and A2-C2). There was no positive staining of collagen type III in normal corneas in the present study (Fig. 7D).

In comparing the proteins, the positive expression of collagen type I in SIS wounds was gradually reduced to normal levels, as shown by immunofluorescence (Fig. $6 \mathrm{C} 1$ and $\mathrm{C} 2$ ), while the $\alpha$-SMA gradually receded to the level of the subepithelial region (Fig. 3C1 and C2). Collagen type III was gradually replaced by collagen type I in the two types of sutured wounds after day 21 (Figs. 6 and 7).

qPCR results. Changes in the expression of four mRNAs from SIS and HMS wounds during the course of corneal wound healing were compared using semi-quantitative PCR. Each of the four mRNA expressions was high on days 14 and 21, but low on months 3 and 6 (Fig. 5). On day 21, the mRNA expression of $\alpha$-SMA and collagen type I reached a peak (Fig. 5A and C). However, the highest expression for vimentin and collagen type III mRNAs was evident on day 14 (Fig. 5B and D). According to statistics, the difference in the mRNA expression of $\alpha$-SMA, vimentin and collagen type III in SIS and HMS wounds was statistically significant $(\mathrm{P}<0.05)$. On day 14 $(\mathrm{P}=0.031)$ and $21(\mathrm{P}=0.05)$ as well as at 6 months $(\mathrm{P}=0.035)$, the HMS wounds produced increaesd $\alpha$-SMA mRNA than the SIS wounds. As for vimentin, the HMS wounds generated increased mRNA than the SIS wounds on day $14(\mathrm{P}=0.023)$. For collagen type III, the HMS wounds produced more mRNA than the SIS wounds on days $14(\mathrm{P}=0.001)$ and $21(\mathrm{P}=0.039)$, and month $6(\mathrm{P}=0.006)$. However, for collagen type $\mathrm{I}$, the mRNA expression between SIS and HMS wounds showed no distinct differences (Fig. 5C). 


\section{Discussion}

The rabbit model of cornea: An opportunity to study the wound healing response in SIS and HMS. While corneal trauma is extremely common, human tissue appropriate for histologic analysis is difficult to obtain. Although patient specimens of penetrating keratoplasty are available, they are often combined with other corneal lesions and cannot be used to investigate normal wound-healing processes.

In the present study, a rabbit model of corneal wound healing was used to overcome these problems. This model correlates general imaging findings with histological and expression analyses at different time-points following a standardized surgical procedure and follow-up. The rabbits were used as the animal models, partly because their eyes are sufficiently large for practical surgical operations. However, the regenerative capacity of the rabbit endothelium is much better than that of humans. Therefore, $1 / 2$ corneal thickness wounds were made in order to compensate for the differences between human and rabbit eyeballs.

The major potential problem of this rabbit model was the use of the same cornea to compare two wounds, as results were potentially confounding between the two wounds. To verify the spacing between two corneal wounds that would preclude interference from one healing process to the other, we initially created numerous corneal wounds and optimized these parameters in the model. It was verified by histological and immunofluorescence assays that no positive cells or proteins were stimulated in the intermediate region between the two selected wounds Additionally, our model design comparing two wounds in the same cornea avoids the discrepancies that are irremediably found using different corneas.

HMS wounds exhibit more fibrosis than SIS wounds in the early healing stages. We used immunofluorescence to mark fibrotic related proteins in the two wounds. The results show that the fluorescence intensities of HMS wounds are stronger than those of the SIS wounds during the early stages. Furthermore, results of qPCR show the mRNAs of these fibrotic proteins are abundantly expressed in HMS wounds during the early stage, supporting these findings. The findings of the images captured (Fig. 1) and the histological sections (Fig. 2) are in agreement with that HMS wounds cause more fibrosis than SIS wounds during the early steps of the woundhealing process.

It has previously been noted that some cells in the anterior stroma can simultaneously express vimentin and $\alpha$-SMA (8). However, our results suggest that, the time of peak for vimentin expression is $\sim 10-14$ days after surgery while that of $\alpha$-SMA is at around day 21 . Thus, it is possible that the transformation of fibroblasts into myofibroblasts lasts 5-7 days in this in vivo model, and accounts for this finding.

Scarring of the cornea is mainly due to improper deposition of ECM components (10). Collagen type III, a main component of early granulation tissue, is gradually decomposed and replaced by normal collagen tissues (6). However, its role in the wound-healing process is incompletely understood (11). Boote suggested that the upregulation of mRNA for collagen type III increased at 2 weeks and decreased at 4 weeks (12). Our results showed that the collagen type III protein and its related mRNA expression reached a peak on day 14 and decreased on day 21. Additionally, the positive expression of collagen type III gradually leveled to a pre-suture point, as indicated by the immunofluorescence. Furthermore, collagen type I, the normally existing collagen in the cornea, may fluctuate in the wound-healing process (6). We have found that the collagen type III may be gradually replaced by collagen type $I$ in the two types of sutured wounds after day 21 . In the remodeling phase, which can last for years, the ECM is remodeled into a structure approaching normal tissue (13).

It is generally accepted that the interaction between epithelial and mesenchymal cells is crucial in abnormal wound repair (7). In our model, HMS evidently breaks the normal interaction of epithelium and matrix. The present results have shown how HMS artificially turned the cornea lamellar stroma out and thus generated more fibrosis than SIS at the beginning of the healing process. Nevertheless, although mattress sutures can produce surface scarring, early removal of these sutures can limit this damage (14). We hypothesize the reason for differences between SIS and HMS not becoming evident after 3 months may be the removal of the stitches at 10 days post-suturing. In our pilot study, the optimal time of stitch removal was on day 10 . Stitches removed at a time earlier than day 9 , resulted in a wound being unable to close adequately, while stitches removed after 10 days were starting to show corneal tissue degradation.

Methodological considerations. Our study was limited by a lack of protein quantitative analyses. This is due to the fact that these fibrotic proteins are not very abundant in a linear scar, and our attempts to identify them by western blot analysis repeatedly failed. However, based on the general imaging findings, histological analyses, immunofluorescence and qPCR results, enough evidence was provided to show a difference in the fibrotic tissues between HMS and SIS during the early stages of wound healing.

In conclusion, wounds in the cornea create scars that induce blurred vision. Good stitching can reduce scar formation. In our rabbit model of cornea wound healing, HMS produced increased fibrosis compared to SIS in the early stages of wound healing, albeit similar outcomes are yielded after 3 months. This observation suggests that it may be beneficial to select SIS over HMS, if only for the more benign initial healing process. A better understanding of wound closure methods may impact corneal wound healing and provide important insights for enhancing the vision of patients undergoing corneal injury suturing.

\section{Acknowledgements}

We are grateful for the technical support of Li Ying, who works for the Vision Repair and Rehabilitation lab of Peking University Third Hospital.

\section{References}

1. Thomas JR and Somenek M: Scar revision review. Arch Facial Plast Surg 14: 162-174, 2012.

2. Henderson J, Sutcliffe M and Gillespie P: Epitendinous suture techniques in extensor tendon repairs - an experimental evaluation. J Hand Surg Am 36: 1968-1973, 2011. 
3. Kan CD and Yang YJ: Double telescopic anastomosis with interrupted suture technique in acute aortic dissection. Ann Thorac Surg 91: 1630-1631, 2011.

4. Fini ME and Stramer BM: How the cornea heals: Cornea-specific repair mechanisms affecting surgical outcomes. Cornea 24 (Suppl 8): S2-S11, 2005.

5. Myrna KE, Pot SA and Murphy CJ: Meet the corneal myofibroblast: The role of myofibroblast transformation in corneal wound healing and pathology. Vet Ophthalmol 12 (Suppl 1): 25-27, 2009.

6. Klingberg F, Hinz B and White ES: The myofibroblast matrix: Implications for tissue repair and fibrosis. J Pathol 229: 298-309, 2013.

7. Weis AJ, Huxlin KR, Callan CL, DeMagistris MA and Hindman HB: Keratocyte apoptosis and not myofibroblast differentiation mark the graft/host interface at early time-points post-DSAEK in a cat model. PLoS One 8: e75623, 2013.

8. Chaurasia SS, Kaur H, de Medeiros FW, Smith SD and Wilson SE: Dynamics of the expression of intermediate filaments vimentin and desmin during myofibroblast differentiation after corneal injury. Exp Eye Res 89: 133-139, 2009.
9. Bargagna-Mohan P, Paranthan RR, Hamza A, Zhan CG, Lee DM, Kim KB, Lau DL, Srinivasan C, Nakayama K, Nakayama KI, et al: Corneal antifibrotic switch identified in genetic and pharmacological deficiency of vimentin. J Biol Chem 287: 989-1006, 2012

10. Karamichos D, Guo XQ, Hutcheon AE and Zieske JD: Human corneal fibrosis: An in vitro model. Invest Ophthalmol Vis Sci 51: 1382-1388, 2010.

11. Volk SW, Wang Y, Mauldin EA, Liechty KW and Adams SL: Diminished type III collagen promotes myofibroblast differentiation and increases scar deposition in cutaneous wound healing. Cells Tissues Organs 194: 25-37, 2011.

12. Boote C, Du Y, Morgan S, Harris J, Kamma-Lorger CS, Hayes S, Lathrop KL, Roh DS, Burrow MK, Hiller J, et al: Quantitative assessment of ultrastructure and light scatter in mouse corneal debridement wounds. Invest Ophthalmol Vis Sci 53: 2786-2795, 2012.

13. Guo S and Dipietro LA: Factors affecting wound healing. J Dent Res 89: 219-229, 2010.

14. Zuber TJ: The mattress sutures: Vertical, horizontal, and corner stitch. Am Fam Physician 66: 2231-2236, 2002. 